\title{
Modelo de análisis espacial multicriterio (AEMC) para el mapeo de servicios ecosistémicos en cuencas forestales del sur de Chile
}

\author{
A multi-criteria model for mapping ecosystem services in forested watersheds, southern Chile
}

\author{
Carlos Esse ${ }^{\text {a* }}$, Paulo Valdivia ${ }^{\text {b }}$ Francisco Encina-Montoya ${ }^{\text {b }}$, Carlos Aguayo b, \\ Marcela Guerrero ${ }^{\text {b }}$, David Figueroa ${ }^{\text {b }}$ \\ *Autor de correspondencia: ${ }^{a}$ Universidad Católica de Temuco, Escuela de Ciencias Forestales, \\ casilla 15-D, Temuco, Chile, cesse@uct.cl

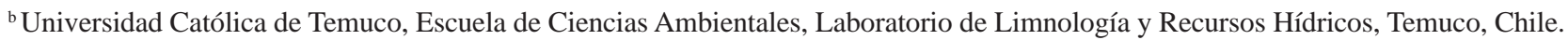

\author{
SUMMARY
}

Ecosystem services provide many benefits to society. These services have been classified into four categories: provisioning services, regulating, cultural and support. One of the major gaps in mapping these services is related to the lack of a standardized methodology that integrates biophysical and perceptual variables derived from the vision of relevant actors in a territory. Multi-criteria spatial analysis (MCSA) is an appropriate methodological framework to integrate this type of information. The present study aims at proposing and applying a methodology of multi-criteria analyses based on GIS tools and homogeneous environmental units (HEU) for mapping ecosystem services in Quepe river basin. Results indicated that provision, regulation and cultural services were significantly associated with the HEU Bosque Nativo. Furthermore, the level of presence of these services was significantly lower in the HEU Plantación and Tierras Agrícolas. We conclude that the method has the advantage of providing ease and flexibility in data spatialization, while collecting perceptual values of a specific service in the sub-basin.

Key words: ecosystem service, multi-criteria evaluation, participatory mapping, homogeneous environmental units.

\section{RESUMEN}

Los servicios ecosistémicos proveen variados beneficios a la sociedad. Dichos servicios han sido clasificados en cuatro categorías: servicios de provisión, regulación, soporte y culturales. Una de las principales brechas en el mapeo social y la espacialización de estos servicios, se relaciona con la ausencia de una metodología que integre variables biofísicas y perceptuales de actores relevantes en un territorio. El análisis espacial multicriterio (AEMC) constituye un marco metodológico apropiado que permite integrar esta información. La presente investigación tuvo como objetivo proponer y aplicar una metodología de análisis multicriterio, basado en herramientas SIG y unidades ambientales homogéneas (UAH) para el mapeo de servicios ecosistémicos en la subcuenca del río Quepe. Los resultados indicaron que los servicios de provisión, regulación y culturales, estuvieron significativamente presentes y asociados a la UAH bosque nativo. Por otro lado, el nivel de presencia de estos servicios fue significativamente menor en las UAH plantaciones y terrenos agrícolas. Se concluye que el método presenta la ventaja de otorgar facilidad y flexibilidad en la espacialización de datos, mientras que recoge de manera sencilla los valores perceptuales de los actores locales y cómo estos perciben el nivel de presencia de un determinado servicio en la subcuenca.

Palabras clave: servicios ecosistémicos, evaluación multicriterio, mapeo participativo, unidades ambientales homogéneas.

\section{INTRODUCCIÓN}

Los servicios ecosistémicos proveen de variados beneficios a la sociedad (Constanza et al. 1997, De Groot et al. 2010). Dichos servicios han sido clasificados en cuatro categorías: servicios de provisión, regulación, culturales y soporte (MEA 2005, Wallace 2007). Los servicios de provisión son aquellos productos que se obtienen de los ecosistemas, incluyendo los recursos genéticos, alimentos y agua potable. Los servicios de regulación son los beneficios que se obtienen a partir de la regulación de procesos ecosistémicos, como la regulación climática, hídrica o la calidad del aire. Adicionalmente, los servicios culturales son todos aquellos beneficios no materiales de los ecosistemas, como el enriquecimiento espiritual, la generación de conocimiento o recreación. Los servicios de soporte se relacionan con aquellos que son necesarios para mantener otros servicios ecosistémicos. Este último se diferencia de las tres primeras clasificaciones dado que los impactos en las personas son indirectos y ocurren en escalas temporales más amplias (MEA 2005).

Uno de los desafíos más relevantes en la evaluación de servicios ecosistémicos se relaciona con determinar la distribución espacial que estos poseen en el territorio. El stock 
de servicios ecosistémicos requiere una representación espacial explícita, para que la toma de decisiones en el manejo de recursos naturales puedan ser ejecutadas de manera informada y eficiente (Crossman et al. 2013). Por ende, la identificación de servicios ecosistémicos requiere de técnicas que permitan abstraer la complejidad del territorio y que contribuyan a identificar los límites de su distribución espacial. En este sentido, los sistemas de información geográfica (SIG) y las técnicas de análisis espacial, como por ejemplo el análisis multicriterio, son herramientas que contribuyen a simplificar la lectura de información territorial, permitiendo generar bases de datos respecto a los atributos que componen el espacio geográfico (De Groot et al. 2002). Dado el creciente interés científico respecto a las técnicas de análisis espacial, estas herramientas han consolidado su posición en la gestión pública de recursos naturales y en la generación de políticas y normativas de regulación ambiental (Brown et al. 2012).

Existen múltiples métodos de modelamiento y mapeo de servicios ecosistémicos, cuyas aproximaciones varían de acuerdo al tipo de servicio, la disponibilidad de datos, la escala espacial de análisis y según el objetivo del estudio (Maes et al. 2012). Sin embargo, una de las brechas más importantes en el mapeo de servicios ecosistémicos tiene relación con un marco metodológico que integre variables biofísicas y la valoración perceptual de los actores locales presentes en el territorio, y así determinar el nivel o valor de importancia de cada servicio. En este sentido, Nahuelhual et al. (2013) indican que los estudios disponibles no incorporan la combinación de las dimensiones ecológicas e institucionales, por lo cual la mayoría de los estudios conocidos ponen énfasis en los elementos físicos del paisaje y rara vez en los elementos sociales, como la percepción o preferencia de las personas en la evaluación del servicio ecosistémico.

$\mathrm{Al}$ respecto, el análisis espacial multicriterio (AEMC) ofrece la posibilidad de definir los estándares metodológicos para el mapeo de servicios ecosistémicos, debido a que dicha técnica es flexible en su forma, permite rescatar la opinión de expertos y actores sociales, la cual es espacializada a través de una plataforma SIG (Malczewski 2006).

La evaluación multicriterio incorpora la opinión o percepción de los actores en las variables y criterios que componen el modelo de evaluación. Los criterios son seleccionados, cuidadosamente, por expertos (evitando la presencia de sesgos), para luego ser ponderados y valorados por los actores locales, mientras que los SIG permiten integrar las variables y sus criterios con atributos geoespaciales (Feick 2010). De esta forma, con la aplicación del AEMC es posible recoger las distintas percepciones de actores respecto a la localización de servicios ecosistémicos, mediante una metodología racional y espacialmente explícita (Fontana et al. 2013).

En la generación de políticas, lineamientos estratégicos y regulaciones normativas de conservación de recursos naturales, se requiere conocer la distribución espacial y el tipo de servicio proporcionado por un área geográfica determinada (Nahuelhual et al. 2013). Ello contribuye a lograr un mejor control sobre las metas de protección ecosistémica a escala general, y optimizar el manejo de la oferta, demanda y flujo de servicios ecosistémicos a un nivel de mayor precisión (Crossman et al. 2013). Por ello, en la toma de decisiones estratégicas de orden territorial, los mapas se constituyen como herramientas fundamentales para comunicar la complejidad de los ecosistemas (Maes et al. 2012, Nahuelhual et al. 2013).

De acuerdo a los antecedentes descritos y considerando que en Chile la aplicación de los SIG y técnicas de análisis espacial en la evaluación ecosistémica es incipiente, este estudio tiene por objetivo proponer un marco metodológico que permita identificar y mapear los servicios ecosistémicos en un área determinada, basado en la definición de unidades ambientales homogéneas (UAH) y análisis espacial multicriterio. Para ello, se aplica la propuesta metodológica en la subcuenca del río Quepe, la cual se inserta en la cuenca del río Imperial, región de La Araucanía, Chile. La evaluación de los servicios ecosistémicos considera los siguientes componentes fundamentales para el análisis: (1) participación de actores relevantes de la cuenca, (2) servicios ecosistémicos asociados al bosque nativo y a los recursos hídricos, y (3) la integración de variables biofísicas del paisaje que se asocian a la provisión de servicios ecosistémicos.

\section{MÉTODOS}

Área de estudio. La cuenca del río Imperial (figura 1) corresponde a la segunda cuenca hidrográfica más importante en términos de superficie en la región de La Araucanía, abarcando un total de $12.696 \mathrm{~km}^{2}$ (DGA 2004). En esta cuenca se distinguen cuatro subunidades: (1) subcuenca del río Quepe con una superficie total de $2.288,5 \mathrm{~km}^{2}$, (2) subcuenca del río Chol-Chol con una superficie total de $5.786,8 \mathrm{~km}^{2}$, (3) subcuenca del río Cautín, con una superficie total de $3.157,5 \mathrm{~km}^{2}$, y (4) subcuenca del río Imperial con una superficie total de $1.463,3 \mathrm{~km}^{2}$, la cual recibe influencia marina (Rivera et al. 2004).

El clima que caracteriza la cuenca es de los tipos templado cálido con influencia mediterránea y templado frío con influencia mediterránea, con precipitaciones medias anuales que alcanzan un total de $1.640 \mathrm{~mm}$, y una temperatura media anual de $11,9{ }^{\circ} \mathrm{C}$ (DGA 2004).

La cuenca presenta elevaciones moderadas, que no sobrepasan los $2.400 \mathrm{~m}$ s.n.m., con pendientes inferiores al $60 \%$. Las máximas altitudes corresponden al volcán Llaima (3.125 m s.n.m.), Sierra Nevada (2.580 m s.n.m.) y volcán Lonquimay (2.822 m s.n.m.), los cuales representan grandes reservorios de agua dulce que contribuyen a la mantención de los caudales de la cuenca (Sánchez y Morales 2000). La Depresión Intermedia presenta planicies y formas geográficas que no superan los $300 \mathrm{~m}$ s.n.m., en cuya área se desarrolla fundamentalmente la actividad 

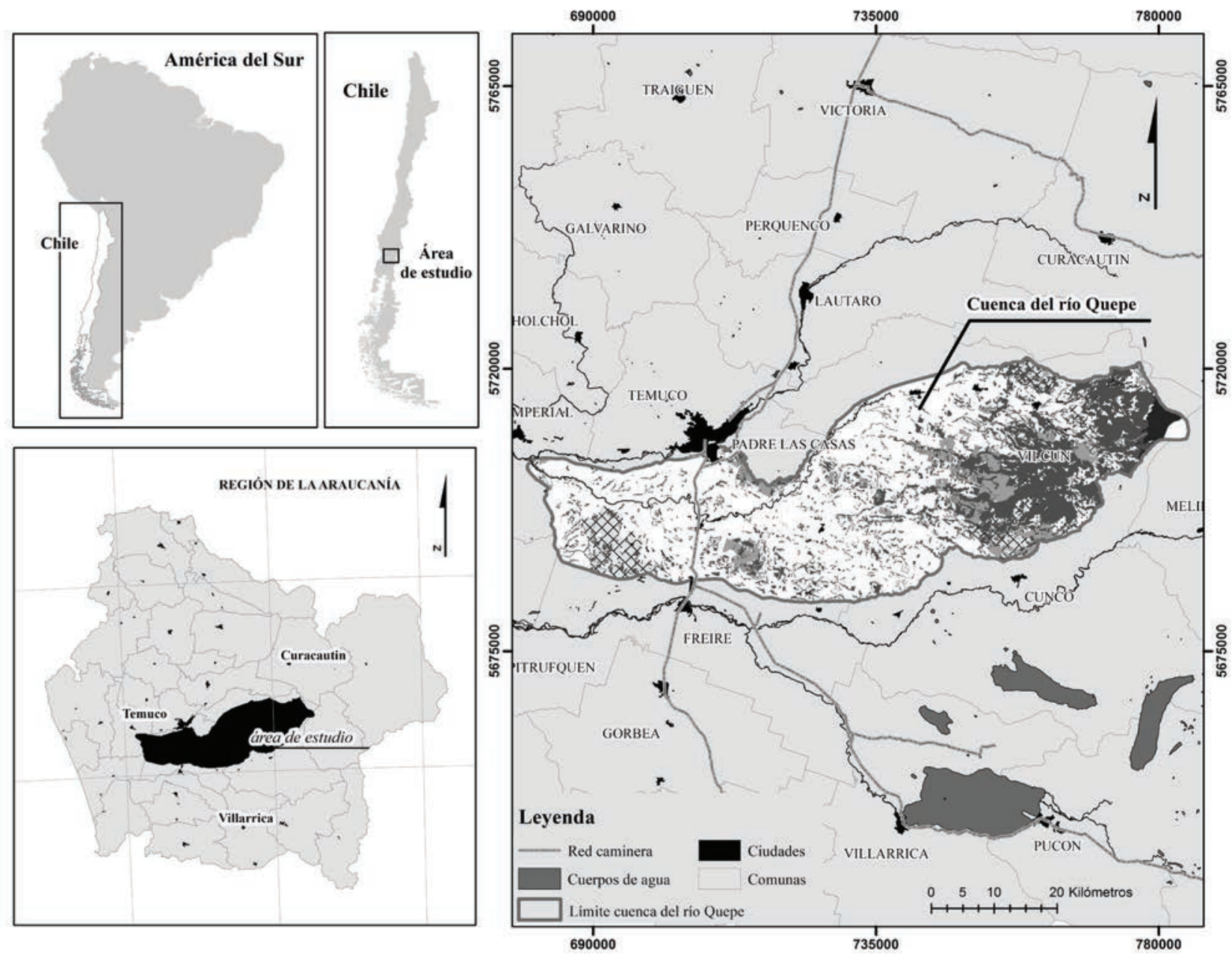

Figura 1. Área de estudio, cuenca del río Quepe.

Study area, in Quepe sub-watershed.

agrícola, y por lo cual los aportes hídricos que se generan en la cuenca son imprescindibles para dicha actividad. El suelo se caracteriza por presentar un origen de cenizas volcánicas (Inceptisols y Andisols) en diferentes etapas de desarrollo. Estos suelos presentan una textura gruesa en profundidad y fina en superficie, con profundidades que pueden llegar hasta más de $1 \mathrm{~m}$ (Luzio et al. 2010).

Unidades ambientales homogéneas. La definición de UAH deriva de un tipo de modelo de planificación física que considera el desarrollo metodológico de procedimientos integrados (Gómez-Orea 1999). Las UAH pueden determinarse en forma simplificada, como resultado de un estudio zonificado de variables concretas, como por ejemplo unidades edafológicas, hidrográficas vegetacionales, entre otras, generando así áreas homogéneas que equivaldrían a las unidades ambientales (Feoli-Boraschi 2009). En este sentido, el paisaje de la subcuenca del río Quepe presenta una alta actividad agrícola y forestal con distintos niveles de productividad, las que permiten definir UAH.
Los usos de suelo predominantes en la subcuenca (cuadro 1) muestran que el uso rotación cultivo-pradera es el que presenta mayor superficie $(56,6 \%)$ respecto del total de la cuenca, seguido por el uso bosque nativo (23,6 \%), mientras que el tercer lugar está representado por el uso plantación (8,1\%). La predominancia de estas actividades en la sub-cuenca es concordante con el porcentaje de representatividad al PIB regional que poseen las actividades silvoagropecuarias, en torno al 10 \% (Banco Central de Chile 2012).

Representación espacial de servicios ecosistémicos en la subcuenca del río Quepe. Basado en la definición y clasificación de servicios ecosistémicos propuestos por Millenium Ecosystem Assessment (MEA 2005) y Haines-Young y Potschin (2013), se realizó el modelamiento y mapeo de servicios ecosistémicos de la subcuenca del río Quepe a través del AEMC, con apoyo de herramientas SIG. El método propuesto se aplicó en tres etapas, las cuales se detallan a continuación (figura 2). 
Cuadro 1. Usos de suelo en la subcuenca del río Quepe.

Land cover distribution in Quepe sub-watershed.

\begin{tabular}{lrc}
\hline \multicolumn{1}{c}{ Usos } & $\begin{array}{c}\text { Superficie } \\
\left(\mathrm{km}^{2}\right)\end{array}$ & $\begin{array}{c}\text { \% del área } \\
\text { total }\end{array}$ \\
\hline Rotación Cultivo-Pradera & 1.295 & 56,60 \\
Bosque Nativo & 541 & 23,60 \\
Plantación & 185 & 8,10 \\
Praderas Perennes & 122 & 5,30 \\
Matorral & 48 & 2,10 \\
Matorral Arborescente & 37 & 1,60 \\
Corridas de Lava y Escoriales & 24 & 1,00 \\
Nieves & 10 & 0,44 \\
Ciudades-Pueblos-Zonas Industrial & 8 & 0,35 \\
Protección & 5 & 0,22 \\
Terrenos de Uso Agrícola & 4 & 0,17 \\
Cajas de Ríos & 2 & 0,09 \\
Lago-Laguna-Embalse-Tranque & 2 & 0,09 \\
Matorral Pradera & 2 & 0,09 \\
Otros Terrenos Húmedos & 1 & 0,04 \\
Ríos & 1 & 0,04 \\
Vegas & 2.288 & 0,04 \\
\hline Total & & \\
\hline
\end{tabular}

En la etapa uno, se definieron las UAH a partir de las cuales se identificaron servicios ecosistémicos. Se aplicó el criterio de máxima superficie productiva por uso actual del suelo, esto es, la identificación de los usos de suelo más importantes asociados a la producción de bienes y servicios ecosistémicos en la subcuenca del río Quepe. Para este análisis se utilizaron las bases cartográficas del Catastro Nacional de los Recursos Vegetacionales de Chile (CONAF et al. 1999) y las coberturas del Centro de Investigación de los Recursos Naturales de Chile (CIREN 2003). La proyección cartográfica correspondió a UTM (Universal Transversal de Mercator) y DatumWGS-84 homologable al proyecto SIRGAS para Chile en Huso 18 sur. Finalmente, las clases de suelo más relevantes en la cuenca (cuadro 1) fueron reagrupadas bajo clasificaciones que permitieron definir las UAH en cinco tipos: (a) bosque nativo, (b) praderas, (c) plantaciones exóticas, (d) recursos hídricos y (e) terrenos agrícolas. El procesamiento de la información espacial mencionada se realizó utilizando el software ArcGis v10.1.

La segunda etapa consideró la aplicación de un procedimiento de mapeo participativo con los actores más relevantes de la subcuenca, considerando como referencia los estudios realizados por Klain y Chan (2012) y Koschke et al. (2012). Esta etapa tuvo como objetivo reflejar los valores que estos actores asignan a los beneficios y servicios ecosistémicos derivados las UAH respectivas.. Esta etapa fue efectuada en dos pasos. En primer lugar, basado en el análisis de expertos y revisión de literatura (De Groot

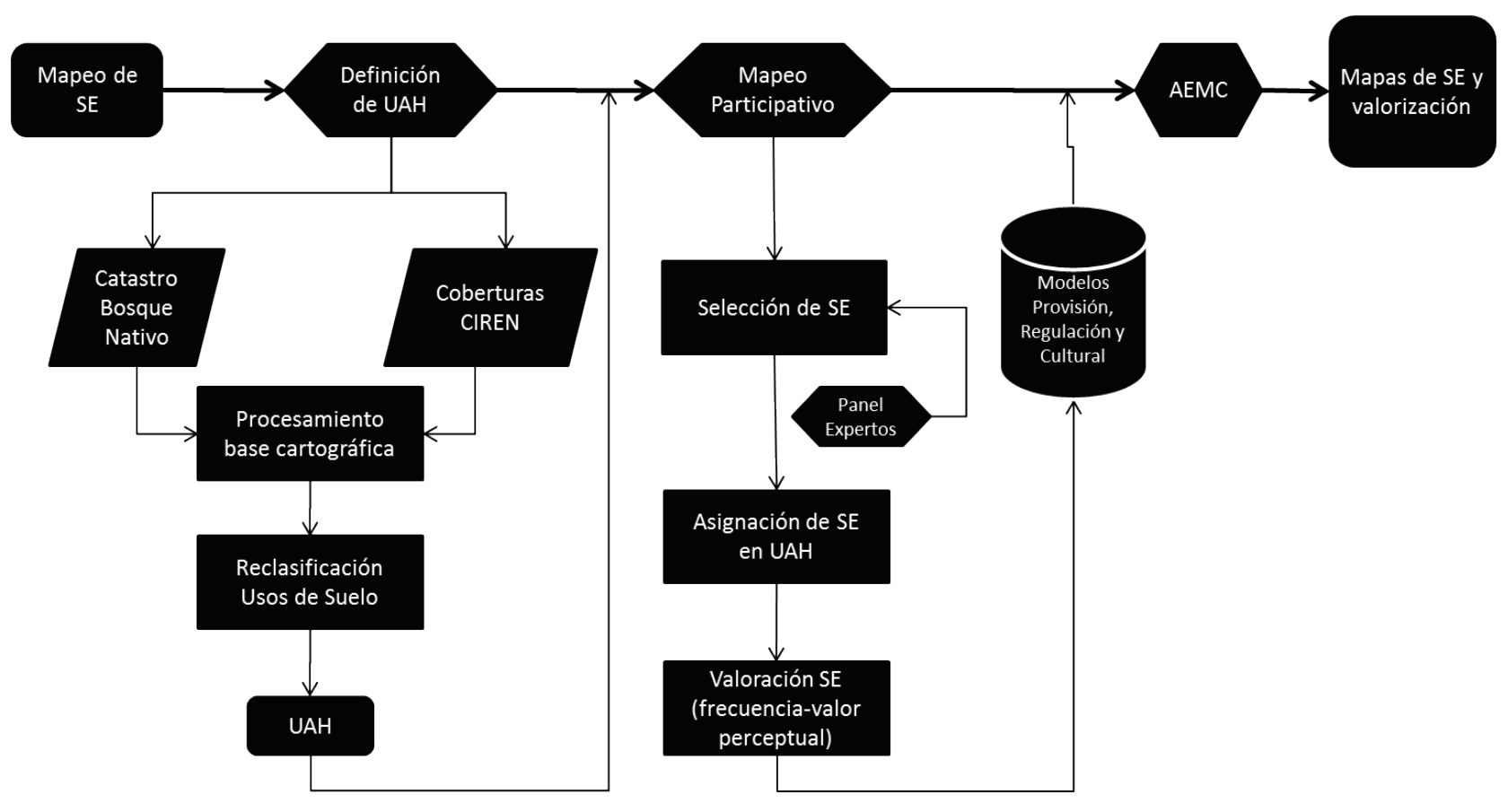

Figura 2. Flujo metodológico para la identificación y mapeo de servicios ecosistémicos.

Methodological flow for identification and mapping of ecosystem services. 
et al. 2002, MEA 2005, Burkhard et al. 2013, HainesYoung y Potschin 2013), se seleccionaron los servicios ecosistémicos más apropiados para cada UAH. El panel de expertos estuvo compuesto por dos ingenieros forestales, un ingeniero en acuicultura, tres biólogos y dos ingenieros ambientales. En segundo lugar, se realizaron reuniones grupales con los actores más relevantes de la cuenca en las cuales a cada participante se le proporcionó un mapa impreso y a color con cada una de las UAH. Se les suministró el listado de servicios, pero sin dar en conocimiento su relación con alguna de las UAH. A cada actor se le solicitó vincular gráfica y espacialmente los servicios a las UAH dentro del mapa. Así, la frecuencia con la cual un determinado servicio apareció asignado en la UAH del mapa, reveló la importancia en dicha unidad espacial, según la apreciación del total de actores locales.. Posteriormente, cada participante asignó un valor a los servicios ecosistémicos identificados en las UAH, utilizando una escala de valorización de 1 (valor bajo), 2 (valor medio) y 3 (valor alto). Lo anterior permitió obtener una segunda medida sobre la importancia perceptual que tiene cada servicio para los actores en la UAH correspondiente, y además posibilitó incorporar dichos valores a los modelos AEMC.

La tercera y última etapa consistió en la aplicación del AEMC en cada UAH, a partir de tres modelos algebraicos ponderados de primer orden, integrando la valoración de los actores locales respecto a los servicios ecosistémicos identificados. Cada modelo se construyó mediante el agrupamiento de servicios ecosistémicos. Los servicios fueron ponderados porcentualmente por el panel de expertos dentro de la ecuación general del modelo, para determinar su jerarquía e importancia dentro de las UAH. Además de esta ponderación, se realizó un ajuste en el valor de cada servicio según su frecuencia en las UAH. El método de AEMC se basó en una adaptación del método original propuesto por Gómez-Orea (1999), cuya metodología considera cuatro modelos (a) valor ecológico, compuesto por atributos referidos a las especies de un área determinada y al valor que adquieren los ecosistemas donde estas especies habitan; (b) valor productivo, compuesto por atributos vinculados a las actividades productivas-económicas que se desarrollan en un territorio; (c) valor socio-cultural, que corresponde a un valor otorgado por la población local a una unidad territorial de servicio; y (d) valor paisajístico, que corresponde al valor otorgado por la población local a una unidad territorial de servicio o uso comunitario. Finalmente, luego de la aplicación del AEMC, se elaboraron mapas que reflejaron la presencia de servicios ecosistémicos en las UAH.

\section{RESULTADOS}

Definición de unidades ambientales homogéneas. La figura 3 muestra las cinco UAH definidas, estas son: (a) unidad terrenos agrícolas, con una superficie de $1.299 \mathrm{~km}^{2}(56,76$ $\%$ de la superficie total) que comprenden cultivos de trigo, avena, papas, entre otros, distribuidos homogéneamente en casi la totalidad de la subcuenca bajo estudio; (b) unidad bosque nativo, que cubre una superficie de $642 \mathrm{~km}^{2}$ (28,05\% de la superficie total) compuestos esencialmente por bosque secundarios de especies del género Nothofagus de los tipos forestales roble-raulí-coigüe y coigüe-raulí-tepa, ubicados en la parte alta de la cuenca; (c) unidad plantaciones exóticas, con una superficie de 174,3 $\mathrm{km}^{2}(7,61 \%$ de la superficie total) conformados por especies de los géneros Pinus y Eucalyptus, concentrada en áreas específicas dentro de la cuenca; (d) unidad praderas, con 123,9 km² de superficie $(5,41 \%$ de la superficie total) compuestas principalmente por praderas agrícolas, ganaderas, matorral abierto y praderas naturales; y (e) unidad recursos hídricos, cuya superficie alcanza los $1,75 \mathrm{~km}^{2}(0,29 \%$ de la superficie total), correspondiente a lagos, lagunas, ríos, cauces de ríos, esteros, vegas, humedales temporales y permanentes.

Mapeo participativo. Se consultaron a 25 actores representativos de la subcuenca del río Quepe, provenientes de servicios públicos, oficinas/direcciones municipales y organizaciones de turismo (cuadro 2). La definición y caracterización de los servicios ecosistémicos, permitió identificar un total de once servicios (cuadro 3).

Los actores identificaron y asignaron servicios ecosistémicos en los mapas que fueron proporcionados, encontrando un total de 252 servicios en la UAH bosque nativo, 187 en la UAH recursos hídricos, 97 para la UAH terrenos agrícolas, 94 en la UAH praderas y 51 en la UAH plantaciones. El cuadro 4, detalla la valoración promedio en escala numérica (1: bajo, 2: medio, 3: alto) que cada uno de los actores realizó respecto a los servicios ecosistémicos identificados en las UAH.

Cuadro 2. Actores consultados en el proceso de identificación y valoración de servicios ecosistémicos de la sub-cuenca del río Quepe.

Stakeholders assessed during the identification and valuation of ecosystem services in Quepe sub-watershed.

\begin{tabular}{lcl}
\hline Organismo & $\begin{array}{c}\text { Número de } \\
\text { actores }\end{array}$ & Comuna \\
\hline MMA & 2 & Temuco \\
CONAF & 1 & Temuco \\
SERNAPESCA & 1 & Temuco \\
SEREMI SALUD & 1 & Temuco \\
Oficinas Municipales & 9 & Vilcún, Temuco \\
Organizaciones de Turismo & 4 & Vilcún \\
ONG & 7 & Padre Las Casas \\
\hline
\end{tabular}

Simbología: Ministerio del Medio Ambiente (MMA), Corporación Nacional Forestal (CONAF), Servicio Nacional de Pesca (SERNAPESCA), Subsecretaria Ministerial de Salud (SEREMI SALUD), Organización No Gubernamental (ONG). 
Cuadro 3. Identificación de los principales servicios ecosistémicos asociados a las UAH. Modificado a partir de MA (2005) y HainesYoung y Potschin (2012). Potschin (2012).

Identification of ecosystem services in the HEU (homogeneous environmental units). Modified from MEA (2005) and Haines-Young and

\begin{tabular}{|c|c|c|}
\hline $\mathrm{N}^{\circ}$ & Servicio ecosistémico & Descripción del servicio ecosistémico \\
\hline 1 & $\begin{array}{l}\text { Plantas y animales, terrestres y } \\
\text { acuáticos }\end{array}$ & $\begin{array}{l}\text { Incluyen las especies nativas y domesticadas utilizadas para nutrición humana (e.g.: Ganado, } \\
\text { cultivos, peces, hierbas, hongos). }\end{array}$ \\
\hline 2 & $\begin{array}{l}\text { Agua para agricultura, con- } \\
\text { sumo humano, subsistencia y } \\
\text { usos industriales }\end{array}$ & $\begin{array}{l}\text { Incluye toda el agua que se extrae de ríos y napas subterráneas para los distintos usos (e.g.: } \\
\text { consumo doméstico, para ganado, pisciculturas, plantas de tratamiento, riego). }\end{array}$ \\
\hline 3 & $\begin{array}{l}\text { Materiales bióticos estructura- } \\
\text { les y energéticos }\end{array}$ & $\begin{array}{l}\text { Incluyen todos aquellos materiales para las construcciones domésticas, ornamentos y obten- } \\
\text { ción de material combustible (e.g.: madera y leña). }\end{array}$ \\
\hline 4 & $\begin{array}{l}\text { Bioremediación, dilución, fil- } \\
\text { tración, y degradación de con- } \\
\text { taminantes }\end{array}$ & $\begin{array}{l}\text { Incluye la capacidad del ambiente biofísico para la regulación de agentes contaminantes y } \\
\text { nocivos, en especial la regulación en la calidad del agua (e.g.: Fitodegradación, fitoabsor- } \\
\text { ción, dilución-degradación de sustancias en cuerpos de agua). }\end{array}$ \\
\hline 5 & Regulación de flujos hídricos & $\begin{array}{l}\text { Involucra la atenuación de crecidas en los flujos hídricos, el control de avenidas o incremen- } \\
\text { tos repentinos de la escorrentía superficial (e.g.: servicios provistos por humedales, franjas } \\
\text { ribereñas, coberturas de bosque nativo). }\end{array}$ \\
\hline 6 & Regulación de la erosión & $\begin{array}{l}\text { Incluye los componentes bióticos y abióticos del ecosistema que controlan los procesos } \\
\text { erosivos del suelo y los deslizamientos en masa (coberturas de bosque nativo, humedales). }\end{array}$ \\
\hline 7 & Regulación climática & $\begin{array}{l}\text { Incluyen los componentes bióticos, especialmente la vegetación que permite el control del } \\
\text { clima local o regional a través del secuestro de carbono (e.g.: Vegetación nativa, humedales, } \\
\text { vegas). }\end{array}$ \\
\hline 8 & $\begin{array}{l}\text { Regulación del ambiente bió- } \\
\text { tico }\end{array}$ & $\begin{array}{l}\text { Abarca la mantención de los ciclos biológicos vitales (e.g.: polinización), del hábitat (inte- } \\
\text { gridad biótica a través de refugios para especies), del pool genético de especies (e.g.: disper- } \\
\text { sión de semillas) y el control de especies exóticas (e.g: mecanismos de control biológicos). }\end{array}$ \\
\hline 9 & Recreación y ecoturismo & $\begin{array}{l}\text { Se relaciona con la experiencia física y sicológica del uso de los ecosistemas en actividades } \\
\text { no extractivas (e.g.: senderismo, nado, observación de fauna, fotografía). }\end{array}$ \\
\hline 10 & $\begin{array}{l}\text { Representación estéticas, espi- } \\
\text { rituales y de no-uso. }\end{array}$ & $\begin{array}{l}\text { Incluyen todas las representaciones intelectuales y significados que el ser humano asigna } \\
\text { a los ecosistemas (e.g.: simbolismos, cosmovisión, lugares o especies sagradas, sentido de } \\
\text { identidad/pertenencia a un lugar, herencia cultural, valores de existencia de las especies } \\
\text { silvestres). }\end{array}$ \\
\hline 11 & Información y conocimiento & $\begin{array}{l}\text { Información y conocimiento } \\
\text { Constituye el acervo histórico, científico y educacional de un determinado grupo humano y } \\
\text { asociado a un espacio geográfico (e.g.: investigación científica, registro de acontecimientos } \\
\text { históricos, establecimientos educacionales). }\end{array}$ \\
\hline
\end{tabular}

En promedio, el mayor valor registrado fue de 2,9 (alto) para el servicio regulación del ambiente biótico, mientras que los servicios bioremediación, dilución, filtración, y degradación de contaminantes recibieron la valoración más baja, con un valor promedio de 2,1 (medio).

Aplicación de modelos para el AEMC. Los servicios ecosistémicos fueron agrupados en categorías los que dieron origen al modelo de AEMC. En total se obtuvieron tres categorías o grupos de servicios ecosistémicos, los cuales fueron de provisión, regulación y cultural (cuadro 5). Los modelos se describen a continuación:

$$
\begin{aligned}
& \text { Provisión }=\mathrm{PA} *(0,175)+\operatorname{AGCH} *(0,65)+\operatorname{MBEE} *(0,175) \\
& \begin{array}{l}
\text { Regulación }=\mathrm{DC} *(0,175)+\mathrm{RFH} *(0,175)+\mathrm{RE} *(0,175)+\mathrm{RC} \\
*(0,175)+\operatorname{RAM} *(0,3)
\end{array}
\end{aligned}
$$

Cultural $=$ REC $*(0,1)+$ RENS $*(0,6)+$ IC $*(0,3)$

Donde: PA corresponde a plantas y animales, terrestres y acuáticos; AGCH es agua para agricultura, consumo humano y usos industriales; MBEE corresponde a materiales bióticos estructurales y energéticos; DC es bioremediación, dilución, filtración, y degradación de contaminantes; RFH corresponde a regulación de flujos hídricos; $\mathrm{RE}$ es regulación de la erosión; RC corresponde a regulación climática; RAM es regulación del ambiente biótico; REC corresponde a recreación y ecoturismo; IC es información y conocimiento; RENS corresponde a representación estéticas, espirituales y de no-uso.

De esta forma, cada grupo de servicios se estableció como un modelo independiente, cuyas variables que lo componen estuvieron definidas por los servicios ecosistémicos correspondientes. Al mismo tiempo, cada variable fue ponderada de acuerdo a su importancia para la UAH a evaluar. 
Cuadro 4. Valores medios de servicios ecosistémicos por grupo de actores en las UAH. 1 = valor bajo; 2 = valor medio; $3=$ valor alto. Ecosystem services values in HEU (homogeneous environmental units). 1 = lowest value; 2 = medium value; 3 = highest value.

\begin{tabular}{|c|c|c|c|c|c|c|c|c|}
\hline \multirow[b]{2}{*}{$\mathrm{N}^{\circ}$} & \multirow[b]{2}{*}{ Servicio ecosistémico } & \multicolumn{7}{|c|}{ Actores locales } \\
\hline & & $\begin{array}{c}\text { Oficinas } \\
\text { Municipales }\end{array}$ & $\begin{array}{c}\text { SEREMI } \\
\text { Salud }\end{array}$ & SERNAPESCA & MMA & CONAF & $\begin{array}{l}\text { Org. } \\
\text { turismo }\end{array}$ & ONG \\
\hline 1 & $\begin{array}{l}\text { Plantas y animales, terrestres y acuá- } \\
\text { ticos }\end{array}$ & 2,3 & 3,0 & 3,0 & 3,0 & 3,0 & 3,0 & 3,0 \\
\hline 2 & $\begin{array}{l}\text { Agua para agricultura, consumo hu- } \\
\text { mano y usos industriales }\end{array}$ & 2,7 & 3,0 & 1,0 & 3,0 & 3,0 & 3,0 & 2,0 \\
\hline 3 & $\begin{array}{l}\text { Materiales bióticos estructurales y } \\
\text { energéticos }\end{array}$ & 2,5 & 1,0 & 3,0 & 3,0 & 3,0 & 2,8 & 3,0 \\
\hline 4 & $\begin{array}{l}\text { Bioremediación, dilución, filtración, } \\
\text { y degradación de contaminantes }\end{array}$ & 2,3 & 2,0 & 1,0 & 2,0 & 2,0 & 2,5 & 2,0 \\
\hline 5 & Regulación de flujos hídricos & 2,6 & 3,0 & 2,0 & 2,5 & 3,0 & 2,8 & 2,0 \\
\hline 6 & Regulación de la erosión & 2,6 & 3,0 & 3,0 & 3,0 & 3,0 & 2,3 & 2,0 \\
\hline 7 & Regulación climática & 2,7 & 3,0 & 3,0 & 3,0 & 3,0 & 2,5 & 3,0 \\
\hline 8 & Regulación del ambiente biótico & 2,7 & 3,0 & 3,0 & 3,0 & 3,0 & 3,0 & 3,0 \\
\hline 9 & Recreación y Ecoturismo & 2,7 & 3,0 & 3,0 & 3,0 & 3,0 & 2,5 & 2,0 \\
\hline 10 & $\begin{array}{l}\text { Representación estéticas, } \\
\text { espirituales y de no-uso, }\end{array}$ & 2,4 & 2,0 & 3,0 & 2,0 & 2,0 & 2,7 & 2,0 \\
\hline 11 & Información y Conocimiento & 2,5 & 2,0 & 1,0 & 2,5 & 2,0 & 2,0 & 3,0 \\
\hline
\end{tabular}

Cuadro 5. Agrupamiento de servicios ecosistémicos según categorías afines para los modelos AEMC.

Ecosystem services grouping according to the main categories for multi criteria models' assembling.

$\mathrm{N}^{\circ} \quad$ Servicio ecosistémico

Categoría de servicios ecosistémicos

a) Plantas y animales, terrestres y acuáticos

1 b) Agua para agricultura, consumo humano y usos industriales

Provisión

c) Materiales bióticos estructurales y energéticos

a) Bioremediación, dilución, filtración, y degradación de contaminantes

b) Regulación de flujos hídricos

2 c) Regulación de la erosión

Regulación

d) Regulación climática

e) Regulación del ambiente biótico

a) Recreación y ecoturismo

3 b) Representación estéticas, espirituales y de no-uso.

Cultural

c) Información y conocimiento

Mapeo de servicios ecosistémicos. La figura 3A muestra el nivel de presencia más alto para el grupo servicios de provisión está determinado por la UAH bosque nativo, mientras que la UAH recursos hídricos alcanzó el segundo valor más alto en la subcuenca. Las UAH terrenos agrícolas y praderas alcanzaron, respectivamente, el tercer y cuarto valor más bajo en toda la subcuenca. La UAH plantaciones alcanzó el valor más bajo de la subcuenca (cuadro 6).
La figura 3B muestra que el valor más alto para el grupo servicios de regulación está determinado por la UAH bosque nativo, seguido de la UAH recursos hídricos. La UAH praderas alcanzó el tercer nivel de importancia respecto de este grupo, mientras que las UAH plantaciones y terrenos agrícola obtuvieron el cuarto y quinto lugar en su valor de importancia, respectivamente. Los servicios ecosistémicos culturales (figura 3C) en la subcuenca del río 
Cuadro 6. Valores de grupos de servicios ecosistémicos en las UAH derivados de la percepción de actores y ponderación de expertos a través de los modelos AEMC.

Ecosystem services values in the HEU derived from the stakeholders perceptions and weighted by the experts in the AEMC models.

\begin{tabular}{lccccc}
\hline \multirow{2}{*}{ Servicios ecosistémicos } & \multicolumn{5}{c}{ UAH } \\
\cline { 2 - 6 } & Terrenos agrícolas & Bosque nativo & Plantaciones & Praderas & Recursos hídricos \\
\hline Provisión & 1,43 & 2,46 & 0,71 & 0,90 & 1,72 \\
Regulación & 0,52 & 2,51 & 0,60 & 0,87 & 1,64 \\
Cultural & 1,06 & 2,01 & 0,65 & 0,88 & 1,96 \\
\hline
\end{tabular}

Quepe, estuvieron mejor representados por la UAH bosque nativo. A su vez, las UAH recursos hídricos y terrenos agrícolas se posicionan en el segundo y tercer lugar de importancia, respectivamente. Finalmente, las UAH praderas y plantaciones poseen, respectivamente, los niveles de presencia más bajo para cada unidad espacial (cuadro 6).

\section{DISCUSIÓN}

Los servicios ecosistémicos mostraron mayor frecuencia (nivel de presencia) en las UAH bosque nativo y recursos hídricos, con un número de asignaciones de 257 y 187 unidades, respectivamente. Esta tendencia en la asignación de servicios se vincula con la relevancia que poseen estos ecosistemas para la sustentabilidad de las diversas actividades en la subcuenca, cuya importancia se deriva de la estrecha relación entre el bosque nativo y la producción de agua. Dicha relación ha sido ampliamente discutida a partir de los escritos originales de Albert (1906) y en investigaciones actuales (Oyarzún et al. 2005, Sherrouse et al. 2011) que dan cuenta del valor del bosque nativo como un ecosistema que mantiene y regula los flujos hidrológicos. Adicionalmente, el alto valor otorgado al bosque nativo y recursos hídricos está determinado por la influencia de la existencia de sitios y espacios de significación cultural mapuche en la subcuenca, que contribuyen al resguardo del acervo cultural de este pueblo originario (Nahuelhual et al. 2013). Por consiguiente, la baja disponibilidad de superficie de bosque nativo de la subcuenca le otorga una alta valoración por parte de las personas que habitan dentro del área y de aquellas que de manera indirecta se vinculan con este recurso (Armesto et al. 1996, Camus 2006).

Por otro lado, la baja asignación de servicios ecosistémicos en las UAH terrenos agrícolas, praderas y plantaciones, con 97, 94 y 51 servicios respectivamente se explica por la sustitución del bosque nativo para habilitación de terrenos en beneficio de las actividades silvoagropecuarias de la subcuenca, las cuales ocasionan un detrimento en la provisión de servicios ecosistémicos que brindan bienestar a las personas (Oyarzún et al. 2005). Desde la perspectiva paisajística la intervención de los sistemas naturales se traduce en una baja valoración del territorio por parte de quienes en forma directa e indirecta hacen uso de los servicios (Little y Lara 2010). Esta percepción se explica en parte por el impacto asociado a la sustitución de bosque nativo por plantaciones forestales, originadas en gran medida por la ley de Fomento Forestal de 1974. En las comunidades rurales del sur de Chile esto se tradujo en la generación de un sentimiento de invasión por parte de la actividad empresarial en el territorio, la cual ha sido asumida como la causante de la pérdida o disminución progresiva de servicios ecosistémicos asociados a las economías de subsistencia locales (Araya 2003).

La actividad agrícola posee una alta representación en el uso del suelo, con un 56,6\% de la superficie total, la que se encuentra asociada a la sección media y baja de la subcuenca. Los sectores altos de la subcuenca se encuentran cubiertos principalmente por vegetación nativa, la cual representa la segunda cobertura de suelo más importante, abarcando un 23,6 \% del total de la subcuenca. De acuerdo al mapeo participativo realizado y el AEMC, la provisión de servicios ecosistémicos como la provisión de agua en cantidad y calidad, o los materiales bióticos estructurales y energéticos se encuentran asociados al bosque nativo comprobado por la alta valoración de esta UAH.

Las técnicas de evaluación multicriterio se han posicionado de manera progresiva en la comunidad científica y en la gestión pública, dado que permiten integrar tanto valores percibidos por las personas como aquellos que son expresados en términos económicos (Malczewski 2006, Sherrouse et al. 2011). En este sentido, los métodos económicos clásicos, se focalizan en realizar una valoración utilitaria de bienes y servicios ecosistémicos. Frecuentemente, estas metodologías presentan dificultades debido a la falta de un mercado adecuado para valorizar aquellos procesos y servicios ecosistémicos de carácter intangible, los cuales se asocian a valores y preferencias percibidos por actores locales (e.g: valor estético, espiritual, histórico) (Chan et al. 2012). Esta percepción está vinculada a los componentes psicológico y cultural de cada individuo y, por tanto, los métodos utilitaristas no permiten capturar el valor del bienestar psicosocial derivados de la relación entre hombre y naturaleza (Kumar y Kumar 2008). Al respecto, Nahuelhual et al. (2013) señalan la importancia fundamental que debe poseer la metodología de espacialización del servicio, la cual debe considerar las percep- 

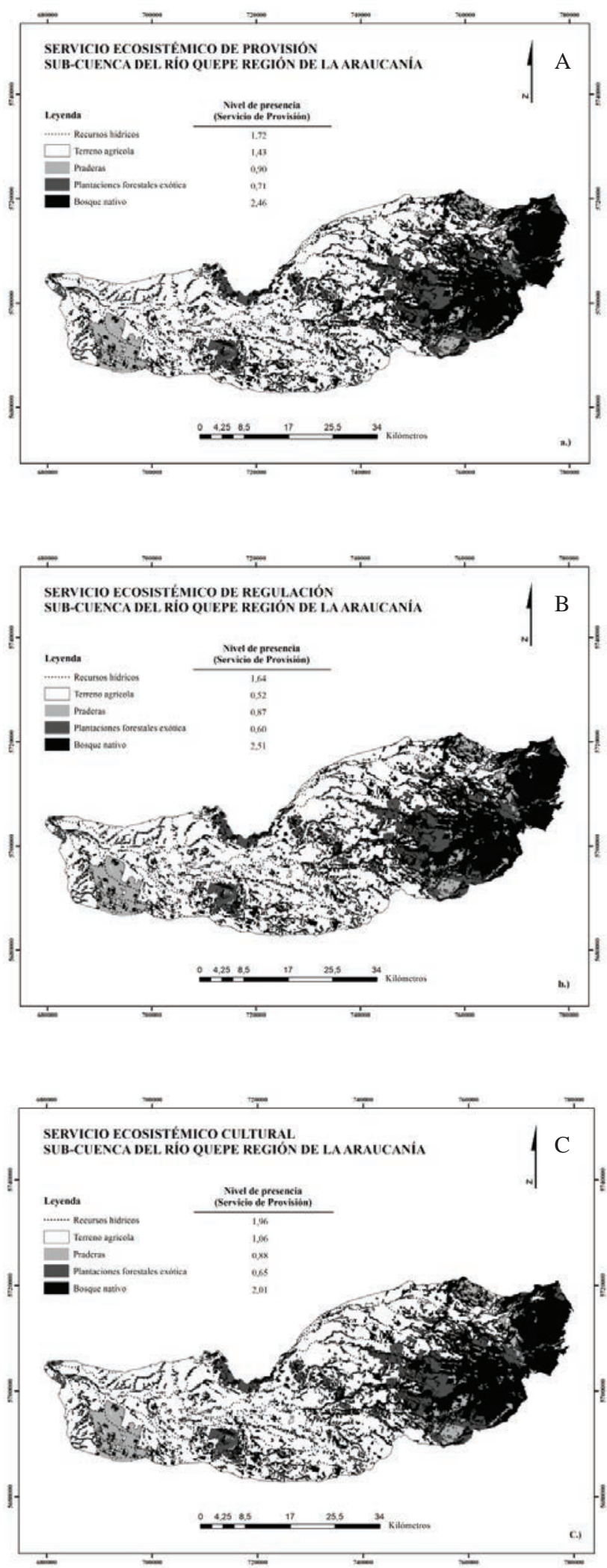

Figura 3. Área de estudio y descripción de los servicios ecosistémicos para cada UAH. En A) servicio de provisión; B) servicio de regulación; C) servicio cultural.

Study area and description of ecosystem services for each HEU. In: A) provision service; B) regulation service; C) cultural service. ciones de los distintos actores, como por ejemplo el panel de expertos, actores locales y autoridades políticas en la identificación de las variables críticas para lograr representaciones válidas y no sesgadas.

La mayor ventaja de incorporar la evaluación multicriterio a las herramientas SIG, es la posibilidad de asignar diversos juicios de valor a los componentes del paisaje y otorgarles proyección espacial (Feick 2010). Dada la facilidad de operación a través de un SIG, los modelos propuestos en el presente trabajo fueron integrados a las UAH, cuyos servicios ecosistémicos recibieron proyección espacial explícita en el paisaje. En definitiva, la valoración del territorio a través de sus atributos, criterios y ponderaciones contribuyeron a establecer la relación entre los componentes del paisaje y los servicios ecosistémicos que mantienen los procesos naturales y las actividades humanas en la subcuenca del río Quepe.

Los modelos propuestos en el presente trabajo constituyen una primera aproximación a la identificación y mapeo de servicios ecosistémicos en cuencas forestales con una fuerte influencia cultural dada por habitantes del pueblo mapuche. Estos permitieron identificar y valorar las características más importantes del territorio que se vinculan con la presencia de servicios ecosistémicos de la subcuenca del río Quepe. La metodología permitió rescatar la percepción de los habitantes del territorio de un modo flexible y, al mismo tiempo, posibilitó de que dicha percepción tuviera una representación espacial (Brown et al. 2012). La inclusión de valores perceptuales y visiones de los actores en un territorio, se considera como uno de los aspectos más determinantes para una adecuada evaluación de servicios ecosistémicos según la literatura actual (Kumar y Kumar 2008, Brown et al. 2012, Chan et al. 2012). Esto se debe a que los servicios son identificados en gran medida a través de las personas que hacen uso de los recursos naturales. Es decir, los servicios son identificados en función de los beneficios que puedan experimentar los individuos y que son derivados de la naturaleza (Kumar y Kumar 2008).

A pesar de las ventajas de la metodología propuesta existen aspectos que deben ser revisados para optimizar su aplicación. Por una parte, el análisis abarcó la consulta a un total de 25 actores de la subcuenca, lo cual pudiera haber afectado la representatividad en las respuestas. Un mayor número de respuestas eventualmente optimizaría el análisis para obtener percepciones que permitan abarcar el mayor rango de visiones de los beneficios y servicios presentes en la subcuenca.

Por otro lado, queda aún por analizar en mayor profundidad la relación entre frecuencia (intensidad) de un servicio ecosistémico y su nivel de importancia. Esto se debe a que los servicios que se identificaron con mayor frecuencia en el presente estudio, fueron aquellos que son más visibles o que se experimentan con mayor intensidad por las personas, como el servicio de provisión de agua. Sin embargo pueden existir otros, como los servicios de 
regulación, que pudieran permanecer inadvertidos por la población debido a la dificultad de su identificación, pues interaccionan entre múltiples procesos ecosistémicos complejos. Adicionalmente, futuros estudios de servicios ecosistémicos debieran considerar tanto el mapeo participativo como también el mapeo por parte de los expertos, y de esta forma superponer ambos tipos de mapas para examinar la congruencia entre los servicios identificados. Este último análisis puede ser complementario al desarrollo de la presente propuesta metodológica, para así incrementar la precisión en la identificación y distribución espacial de servicios ecosistémicos.

\section{CONCLUSIONES}

La identificación de servicios ecosistémicos y sus valores en la presente investigación, constituye una primera aproximación al uso de la tecnología SIG, como apoyo a la construcción de un modelo conceptual alfanumérico que permite la espacialización de las distintas percepciones que poseen los actores claves de un territorio sobre su entorno. Dicho modelo resultó ser apropiado, lográndose una buena representación espacial de la información territorial, lo cual facilita la identificación y posterior mapeo de los servicios ecosistémicos existentes en una cuenca forestal.

Los resultados del estudio muestran una propuesta metodológica, de fácil aplicación para el mapeo de los servicios ecosistémicos, la cual puede ser considerada en otros estudios similares, contribuyendo a la definición de un método, cuya base conceptual no es desconocida en los estudios del medio físico y la ordenación del territorio.

\section{AGRADECIMIENTOS}

Los autores agradecen al Fondo de Investigación en Bosque Nativo administrado por la Corporación Nacional Forestal (CONAF). Además, la participación de los distintos municipios y sus departamentos: Ilustre Municipalidad de Vilcún, Ilustre Municipalidad de Padre las Casas, Ilustre Municipalidad de Temuco, Ilustre Municipalidad de Imperial e Ilustre Municipalidad de Freire. Finalmente, los autores agradecen a la unidad de investigación y la Facultad de Recursos Naturales de la Universidad Católica de Temuco.

\section{REFERENCIAS}

Albert F. 1906. Los servicios de aguas y bosques. Santiago, Chile. Sección de Aguas y Bosques del Ministerio de Industria. 41 p.

Armesto JJ, M Arroyo, C Villagrán. 1996. Ecología de los Bosques Nativos de Chile. Santiago, Chile. Editorial Universitaria. $470 \mathrm{p}$.

Araya J. 2003. Efectos de la actividad forestal en la población indígena mapuche. La invasión de las plantaciones forestales en Chile. Santiago, Chile. Observatorio Latinoamericano de Conflictos Ambientales. 12 p.

Banco Central de Chile. 2012. Cuentas Nacionales de Chile. Evo- lución de la actividad económica en el año 2012. Santiago, Chile. Banco Central de Chile. 4 p.

Brown G, J Montag, K Lyon. 2012. Public participation GIS: a method for identifying ecosystem services. Society and $\mathrm{Na}$ tural Resources 25 (7): 633-651.

Burkhard B, N Crosman, S Nedkov, K Petz, R Alkemade. 2013. Mapping and modelling ecosystem services for science, policy and practice. Ecosystem Services 4: 1-3.

Camus P. 2006. Ambiente, bosques y gestión forestal en Chile.1541-2005. Colección Sociedad y Cultura, Central de Investigaciones Diego Barros Arana. Santiago, Chile. LOM Ediciones. 374 p.

Chan K, T Satterfield, J Goldstein. 2012. Rethinking ecosystem services to better address and navigate cultural values. Ecological Economics 74: 8-18.

CIREN (Centro de Información de Recursos Naturales, CL).2003. Estudio Agrológico de la X Región. Tomos I y II. Santiago, Chile. CIREN. 412 p.

CONAF (Corporación Nacional Forestal, CL), CONAMA (Comisión Nacional del Medioambiente, CL), BIRF (Banco Internacional de Reconstrucción y Fomento, US), Universidad Austral de Chile, Pontificia Universidad Católica de Chile, Universidad Católica de Temuco. 1999. Proyecto Catastro y Evaluación de los Recursos Vegetacionales Nativos de Chile, Santiago, Chile. Disponible en www.conaf.cl

Costanza R, R D’arge, R De Groot, S Farberk, M Grasso, B Hannon, K Limburg, S Naeem, R O’neill, J Paruelo, R Raskin, P Suttonkk, M Van Den Belt. 1997. The value of the world's ecosystem services and natural capital. Nature 387: 253260.

Crossman N, B Burkhard, S Nedkov, L Willemen, K Petz, I Palomo, E Drakou, B Martín-López, T McPhearson, K Boyanova, R Alkemade, B Egoh, M Dunbar, J Maes. 2013. A blueprint for mapping and modelling ecosystem services. Ecosystem Services 4: 4-14.

De Groot R, M Wilson, R Boumans. 2002. A typology for the classification, description and valuation of ecosystem functions, goods and services. Ecological Economics 41: 393-408.

DGA (Dirección General de Aguas, CL). 2004. Diagnóstico y clasificación de los cursos y cuerpos de agua según objetivos de calidad. Cuenca del río Imperial. Santiago, Chile. Ministerio de Obras Públicas. 137 p.

Feick R. 2010. Spatialmulticriteria evaluation. In Warf B. ed. Encyclopedia of geography. Thousand Oaks, Ca, USA. SAGE Publications. p. 2656-2658.

Feoli-Boraschi S. 2009. Corredores biológicos: una estrategia de conservación en el manejo de cuencas hidrográficas. Kurú: Revista Forestal Mesoamericana 6(17): 1-5.

Fontana V, Radtkeb A, Bossi Fedrigottib V, Tappeinera U, Tasserc E, Zerbeb S, Buchholzd T. 2013. Comparing land-use alternatives: Using the ecosystem services concept to define a multi-criteria decision analysis. Ecological Economics 93: 128-136.

Gómez-Orea D. 1999. Evaluación de impacto ambiental. Un instrumento preventivo para la gestión ambiental. Madrid, España. Ediciones Mundi-Prensa. Editorial Agrícola Española. $701 \mathrm{p}$.

Haines-Yong R, M Potschin. 2013. CICES V4.3-Revised report prepared following consultation on CICES Version 4, August-December 2012. EEA Framework Contract No EEA/ 
IEA/09/003. Nottingham, UK. University of Nottingham. $32 \mathrm{p}$.

Klain SC, K Chan. 2012. Navigating coast values: Participatory mapping of ecosystem services for spatial planning. Ecological Economics 82: 104-113.

Koschke L, C Fürst, S Frank, F Makeschin. 2012. A multi-criteria approach for an integrated land-cover-based assessment of ecosystem services provision for planning support. Ecological Indicators 21:54-66.

Kumar M, P Kumar. 2008. Valuation of the ecosystem services: a psycho-cultural perspective. Ecological Economics 64(4): 808-819.

Little C, A Lara. 2010. Restauración ecológica para aumentar la provisión de agua como un servicio ecosistémico en cuencas forestales del centro-sur de Chile. Bosque (3) 175-178.

Luzio W, M Casanova, O Seguel. 2010. Suelos de la Zona Desértica. In Luzio W ed. Suelos de Chile. Santiago, Chile. Departamento de Ingeniería y Suelos. Facultad de Ciencias Agronómicas. Universidad de Chile. p. 35-75

Maes J, B Egoh, L Willemen, C Liquete, P Vihervaara, JP Schägner, B Grizzetti, EG Drakou, A Lanotte, G Zulian, F Bouraoui, ML Paracchini, L Braat, G Bidoglio. 2012. Mapping ecosystem services for policy support and decision making in the European Union. Ecosystem Services 1: 31-39.

Malczewski J. 2006. GIS-based multicriteria decision analysis: A survey of the literature International Journal of Geographical Information Science 20(7): 249-268.
MEA (Millennium Ecosystem Assessment). 2005. Ecosystems and human well-being: Policy responses. Washington, DC, USA. Island Press. 621 p.

METI (Ministry of Economy, Trade, and Industry, JP), NASA (National Aeronautics and Space Administration, US). 2011. ASTER Global Digital Elevation Model (GDEM) v.2. Consultado 18 ene. 2014. Disponible en http://www. jspacesystems.or.jp/ersdac/GDEM/E/.

Nahuelhual L, A Carmona, P Lozada, A Jaramillo, M Aguayo. 2013. Mapping recreation and ecotourism as a cultural Ecosystem service: An application at the local level in Southern Chile. Applied Geography 40:71-82. DOI: 10.1016/j.apgeog.2012.12.004.

Oyarzún C, L Nahuelhual, D Núñez. 2005. Los servicios ecosistémicos del bosque templado lluvioso: producción de agua y su valoración económica. Revista Ambiente y Desarrollo 20(3)-21(1): 88-95.

Rivera N, F Encina, A Muñoz-Pedreros, P Mejias. 2004. La Calidad de las Aguas en los Ríos Cautín e Imperial, IX RegiónChile. Información tecnológica .15 (5): 89-101.

Sánchez A, R Morales. 2000. Las regiones de Chile. Santiago, Chile. Universitaria. 262 p.

Sherrouse BC, JM Clement, DJ Semmens. 2011. A GIS application for assessing, mapping, and quantifying the social values of ecosystem services: Applied Geography 31 (2): 748-760.

Wallace KJ. 2007. Classification of ecosystem services: problems and solutions. Biological Conservation 139 (3-4): 235-246.

Recibido: 10.03 .14

Aceptado: 01.08.14 
